traité, et qui sont absolument sans valeur artistique aucune . . . et qu'il existe des centaines d'églises en Europe dont le type doit être résolument écarté a priori en pays de mission.' Après avoir formulé des directives générales concernant l'utilisation de l'art indigène dans les domaines de l'architecture, de l'ameublement, de l'iconographie et même du théâtre religieux, il arrive à des conclusions pratiques, qu'il est intéressant de relever ici: 'Étant donnée l'utilité indiscutable de respecter autant que faire se peut les manifestations du génie de tous les peuples, même les plus primitifs, (a) il faut que les missions accordent dès à présent une attention sympathique à l'art indigène. (b) Pour cela il faut des spécialistes: le dilettantisme ne suffit pas, il faut des gens compétents, au goût sûr, et ayant une préparation adéquate. Ce n'est que grâce à des missionnaires formés de la sorte qu'on résoudra le problème de l'art indigène, il faut donc souhaiter vivement leur apparition. (c) Il semble qu'une des premières choses à faire ensuite serait de composer un manuel d'art indigène, d'art chrétien, d'art congolais christianisé. Ce manuel devrait rappeler à chaque page qu'un art qui vit de la foi et l'exprime fidèlement est un des plus puissants moyens de conquête que les missionnaires aient à leur disposition pour la plus grande gloire de Dieu. Dès ses premières directives, ce manuel devrait orienter ceux qui le prendraient pour guide à voir avec des yeux d'indigène chrétien et à ne pas copier sans plus les formes traditionnelles et les méthodes de l'Europe, quitte à les exprimer en matériaux indigènes. Le manuel devrait prendre soin de favoriser et de ne pas dénaturer l'éclosion normale des possibilités artistiques indigènes; il devrait provoquer une sympathique et intelligente compréhension de l'art indigène et diffuser celle-ci largement parmi les civilisateurs.' (Communication du PROFESSEUR N. DE CLEENE.)

\title{
Mission du Directeur en Afrique Occidentale Frangaise.
}

Ainsr que nous l'avons indiqué dans notre précédent numéro, l'un de nos Directeurs, le Professeur H. Labouret, a accompli en Afrique Occidentale Française, pour le compte de son Gouvernement, une mission de cinq mois au cours de laquelle il a visité et étudié les populations du Sénégal, de la Guinée, de la Côte d'Ivoire et du Soudan. Le but principal de ce déplacement était d'effectuer des recherches d'ordre ethnologique sur l'agriculture, le travail, les niveaux de vie et l'alimentation des indigènes dans les territoires observés. Grâce à l'aide de l'administration locale, notre Directeur a pu réunir sur ces importantes questions un matériel très important qu'il va ordonner et présenter dans plusieurs rapports destinés aux Congrès internationaux de l'Exposition de 1937 à Paris.

Le Professeur Labouret a réussi en outre à compléter les collections ethnographiques du Trocadéro et à recueillir une documentation linguistique sur les idiomes en usage dans les pays qu'il a traversés. En particulier il a 
rapporté des textes en Peul du Fouta Djallon et dans les divers dialectes mandingues du Sud. Par ailleurs, le Professeur Labouret a établi les contacts personnels avec les Gouverneurs et les Administrateurs des régions dans lesquelles il a séjourné, il leur a fait connaître l'action et les buts de l'Institut, pour lesquels ces fonctionnaires ont marqué un vif intérêt.

Enfin, au cours de ce voyage, des films intéressants ont été enrégistrés sur la vie indigène et les techniques de la zône soudanaise et de la région sylvestre. Ainsi qu'il l'avait fait précédemment, M. Labouret avait tenu à emmener avec lui un de ses élèves, M. J. J. Villandre, diplômé de l'École Coloniale et de l'École Nationale des Langues Orientales Vivantes.

\section{Etudes des besoins des indigènes dans les colonies françaises.}

LE Gouvernement Français vient de décider la création d'un Comité chargé d'examiner les aspirations et les divers besoins des indigènes dans les colonies, pays de protectorat et territoires sous Mandat placés sous l'autorité de la France. Cet organisme sera présidé par M. Guernut, ancien Ministre; il comprendra trois sénateurs, six députés, et vingt autres membres choisis parmi des personnes s'étant occupées des diverses possessions et qui seront appelées à donner leurs avis en qualité d'experts. Cette Commission sera divisée en trois sous-Commissions: la première pour l'Afrique du Nord, la seconde pour l'Afrique Tropicale, Madagascar, les Antilles et la Guyane, la troisième pour l'Indochine et les terres françaises du Pacifique. Chacune de ces trois sections sera dirigée par un Secrétaire Général. Le Gouvernement de la République a décidé de confier ces fonctions, en ce qui touche la deuxième section, à notre Directeur, M. le Professeur Labouret.

Bien que le programme de recherches à poursuivre par cet organisme ne soit pas encore arrêté, il est probable qu'il comprendra des investigations sur la participation des indigènes à l'administration de leur pays, des recherches sur les conditions du travail, les niveaux de vie, l'alimentation, l'hygiène, l'assistance médicale, l'enseignement. Ce Comité examinera également les problèmes de la production et de la main-d'œuvre.

\section{L'Exposition Internationale de Paris, 1937.}

LA plus grande activité règne actuellement sur les chantiers de l'Exposition Internationale de Paris en I937. On sait qu'en cette occasion de nombreux Congrès, sans doute plusieurs centaines, seront tenus à Paris. Parmi ceux qui peuvent intéresser les membres de notre Institut, nous signalerons celui de la Recherche Scientifique, qui se divisera lui-même en de nombreuses branches formant des sections particulières. L'une de celles-ci sera consacrée à l'ethnologie; elle comprendra naturellement des divisions que nous ferons connaître ultérieurement. 